\title{
Back Pain Following Thoracic Epidural Placement-Now What?
}

\author{
Gregory Rose*1, J.Thomas McLarney ${ }^{1}$ \\ Correspondence: glrose0@email.uky.edu \\ 'Department of Anesthesiology, University of Kentucky college of Medicine, Kentucky, USA.
}

\begin{abstract}
We describe a case of severe back pain following thoracic epidural placement. We discuss the differential for such a case, and the management as well.
\end{abstract}

One of the most feared complications of epidural placement is epidural hematoma. Back pain following epidural catheter placement can have several causes, from those of a trivial nature to those that are a true medical emergency, and can range in degree from mildly sore to excruciating in nature. We present a case of severe back pain shortly following epidural catheter placement that mimicked epidural hematoma but was not.

\section{Case}

A 66 year old 80 kilogram white male with non-small cell carcinoma of the lung was scheduled for a thoracotomy with excision of the mass. His past medical history was significant for a 100 pack-year cigarette smoking history, hypertension, and osteoarthritis. He took no antithrombotic or antiplatelet drugs, and had no history of bleeding diatheses or easy bruising. His coagulation studies were within normal limits, specifically a platelet count of 428, PT/INR 10.8/1.0, and PTT of 29. After sedation with 2 milligrams of midazolam administered intravenously, an epidural catheter was placed at the level of T6/T7 via a midline insertion for postoperative pain control preoperatively in the holding room. The placement itself was unremarkable, with loss of resistance (with air) at 7 centimeters, and the catheter was easily threaded to 12 centimeters at the skin. A 3 milliliter test dose of 1.5\% lidocaine with epinephrine 1:200,000 was negative. The patient tolerated the procedure well.

The patient's entrance into the operating room was delayed for a time. Three and a half hours later, the holding room staff advised us that the patient was complaining of severe back pain. The patient was found to be sitting on the side of the bed, asking for the epidural catheter to be removed. He said lying on the bed was too painful. He related that his pain was $10 / 10$, sharp in nature, in the immediate area of the epidural catheter. He also related that dorsiflexion of his feet worsened the pain. There was slight radiation of the pain superiorly. His strength in all extremities was normal, and his patellar reflexes were intact. The epidural site was normal in appearance, and no blood or clear fluid was obtained upon aspiration of the catheter.

A stat magnetic resonance imaging (MRI) was ordered, and neurosurgery was alerted to be on standby in case an epidural hematoma was discovered on imaging. The MRI showed no evidence of epidural hematoma, and the epidural catheter was visible in the epidural space; there was an incidental finding of a hemangioma in the body of the T4 vertebra. Furthermore, there was no significant disc disease or osteoarthritic changes.

The catheter was removed following the MRI; over the next few hours, his pain gradually decreased. The patient was treated with opiate analgesics overnight, and the surgery was postponed. The patient underwent his planned thoracotomy the next day, and his postoperative pain was managed by intravenous, and then later, oral narcotics.

\section{Discussion}

Incidence of hematoma development after epidural catheter is variable depending on the study and the time period of the study. However the American Society of Regional Anesthesia (ASRA), in their guidelines for patients receiving antithrombotic therapy, quotes an incidence of approximately 1:150,000 [1]. Risk factors for epidural hematoma after epidural catheter placement include antithrombotic and antiplatelet therapy, traumatic placement, and a history of coagulation abnormality. The hematoma can develop at any time after the procedure, whether a catheter is placed or not, and can happen following catheter removal $[2,3]$. One study reports that $50 \%$ of hematomas occur after catheter removal [4]. Infusions of local anesthetics into the catheter postoperatively can mask the symptoms [5].

The presentation of an epidural hematoma can vary from being painless to causing severe pain; the pain may be sharp, radiating back pain that is often severe. Sensory and motor deficits along with urinary retention do not have to be present at the same time the pain develops $[5,6]$. Weakness may be erroneously thought to be from local anesthetic infusion. Any sudden increase in weakness during an epidural infusion should be investigated as potentially being due to a hematoma. The hematoma acts as a space-occupying lesion, causing cord compression and ischemia. It is a true neurological emergency, and evacuation of the hematoma needs to be done as soon as possible after establishment of a diagnosis. Magnetic resonance imaging is the standard means of diagnosis [3] but can also be diagnosed by CT 
Rose. Journal of Anesthesiology and Clinical Science 2012,

http://www.hoajonline.com/journals/pdf/2049-9752-1-2.pdf

doi: $10.7243 / 2049-9752-1-2$

scan. The sooner the decompression is done the better [3], obviously, but if it is done within 8 hours the outlook for return of function is good. In our patient, the complaint of severe back pain 3 hours or so after epidural placement was certainly consistent with the possibility of epidural hematoma formation, despite the fact that his coagulation studies were normal, the lack of antithrombotic therapy, and atraumatic catheter placement. At the time of examination, the patient did not have any neurologic symptoms accompanying the severe back pain, but nevertheless we chose not to wait for symptoms to develop before obtaining proper imaging studies. It is important to realize that if the possibility of epidural hematoma exists, proper diagnostic studies need to be performed immediately; waiting until the scheduled surgery is finished would be detrimental to the extreme and indefensible medicolegally.

The vertebral hemangioma at T4 was an incidental finding on this patient. A vertebral hemangioma is a benign tumor of the vertebrae, and is in fact the most common tumor of the vertebrae, incidentally found in $10-12 \%$ of autopsies. They often are asymptomatic, but can cause back pain. Rarely do they cause cord compression. When they do become symptomatic, resection is the primary treatment. We can find no reports of vertebral hemangiomas complicating epidural catheter placement in the literature.

We are unsure exactly what caused our patient to develop his pain after epidural placement. It is possible that the catheter may have impinged and irritated nerve roots in the area of $\mathrm{T} 4$, radiating towards the vertebral body hemangioma at that level. As well, the circumstances could have been coincidental to the catheter placement. Positioning of a patient during catheter placement could cause spasms in the paravertebral musculature as well [7].

Severe back pain following epidural injection of 2-chloroprocaine [8] has been reported, but our patient received no other local anesthetic than the test dose of lidocaine. Transient neurologic symptoms (TNS) following lidocaine intrathecally are of course well known, but there have also been cases of TNS reported after epidural injection of local anesthetic. However these reports involved infusions or repeat boluses of epidural lidocaine or bupivicaine after a test dose [9].

\section{Conclusion}

we wish to stress the importance of recognizing the possibility of epidural hematoma any time a patient complains of back pain after epidural catheter placement or removal. Epidural hematoma should be considered a neurologic emergency and should be evaluated and treated as soon as it is suspected.

\section{Article History}

Editor: Andra D. DiStefano, Maryland School of Medicine, USA. Received: 23-Jan-2012 Revised: 08-Feb-2012 Accepted: 15-Feb-2012

Published: 12-Mar-2012

\section{Competing interests}

The authors declare that they have no competing interests.

\section{References}

1. Horlocker, T. T. et al. Regional anesthesia in the patient receiving antithrombotic or thrombolytic therapy: American Society of Regional Anesthesia and Pain Medicine Evidence-Based Guidelines (Third Edition). Reg Anesth Pain Med 35, 64-101

2. Varitimidis, S. E. et al. Epidural hematoma secondary to removal of an epidural catheter after a total knee replacement. A case report. J Bone Joint Surg Am 89, 2048-2050

3. Meikle, J. et al. Detection and management of epidural haematomas related to anaesthesia in the UK: a national survey of current practice. $\mathrm{Br}$ J Anaesth 101, 400-404

4. Vandermeulen, E. et al. Anticoagulants and Spinal-Epidural Analgesia. Anesth Analg 79, 1165-77.

5. Bedforth, N. et al. Haematoma and Abscess after epidural Analgesia. British Journal of Anaesthesia 101, 291-3.

6. Gilbert, A. et al. Epidural hematoma after outpatient epidural anesthesia. Anesth Analg 94, 77-78, table of contents

7. Fox, M. W. et al. The natural history and management of symptomatic and asymptomatic vertebral hemangiomas. J Neurosurg 78, 36-45

8. Stevens, R. A. et al. Back pain after epidural anesthesia with chloroprocaine. Anesthesiology 78, 492-497

9. Markey, J. R. et al. Transient neurologic symptoms after epidural analgesia. Anesth Analg 90, 437-439

\section{Citation:}

Rose G and McLarney J T: Back Pain Following Thoracic Epidural Placement-Now What? journal of Anesthesiology and Clinical Science 2012, 1:2. http://www.hoajonline.com/jacs/2049-9752/1/2 